\title{
Remarques sur l'intégration des fonctions \\ $a^{n} \cos \alpha d a, \alpha^{n} \sin \alpha d a$. \\ Par M. Edouard Collignon, \\ Inopecteur général des Ponts et Chaussées en retraite.
}

Lorsqu' on intègre successivement les fonctions

$\cos \alpha d a, \alpha \cos \alpha d \alpha, a^{2} \cos \alpha d \alpha, \alpha^{3} \cos \alpha d a \ldots \ldots$,

où le cosinus de l'arc a est multiplié par une puissance à exposant entier de l'arc lui-même,

on reconnaît que les intégrales sont toutes comprises dans la formule

$$
P \sin \alpha+Q \cos \alpha \text {, }
$$

où $\mathbf{P}$ et $\mathbf{Q}$ représentent des polynomes entiers en $\alpha$ que l'on détermine dans chaque cas particulier.

On a en effet

$$
\begin{aligned}
& \int \cos \alpha d \alpha=\sin \alpha \\
& \int a \cos a d a=a \sin \alpha+\cos a \\
& \int a^{2} \cos \alpha d a=\left(a^{2}-2\right) \sin a+2 a \cos a \\
& \ldots \ldots \ldots \ldots \ldots \ldots \ldots \ldots \ldots \ldots
\end{aligned}
$$

L'intégration de $\sin a d a, a \sin \alpha d a, a^{2} \sin \alpha d a, \ldots .$. donnerait lieu à des relations analogues.

$$
\$ 1
$$

Cherchons d'abord la loi de formation des polynomes $\mathbf{P}$ et $\mathbf{Q}$, et posons d'une manière générale

$$
\text { (1) } \int a^{n} \cos a d a=P_{n} \sin a+Q_{n} \cos \alpha \text {, }
$$

en mettant en évidence l'exposant $n$ dont dépend la forme des polynomes cherchés. 
Différentions ; nous devrons avoir identiquement

$$
a^{n} \cos \alpha=\left(\mathrm{P}_{n}+\frac{d \mathrm{Q}_{n}}{d a}\right) \cos \alpha+\left(\frac{d \mathrm{P}_{n}}{d a}-\mathrm{Q}_{n}\right) \sin \alpha,
$$

et par conséquent nous devrons poser

$$
\text { (2) }\left\{\begin{array}{l}
\frac{d \mathrm{P}_{n}}{d \alpha}-\mathrm{Q}_{n}=0, \\
\frac{d \mathrm{Q}_{n}}{d \alpha}+\mathrm{P}_{n}=\alpha^{n} .
\end{array}\right.
$$

La première équation montre que $Q_{n}$ est la dérivée de $P_{n}$ par rapport a $a$. Eliminons $Q_{n}$ entre les deux équations (2). Il viendra

$$
\text { (3) } \frac{d^{2} P_{n}}{d a^{2}}+P_{n}=a^{n}
$$

équation différentielle du second ordre, dont nous devrons prendre seulement la solution particulière dans laquelle $P_{n}$ est un polynome entier en $a$. Posons, en appelant $A_{1}, A_{2}, A_{3}, \ldots \ldots$ des coefficients indéterminés,

$$
P_{n}=a^{n}+A_{1} a^{n-1}+A_{2} a^{n-2}+\ldots+A_{n-2} a^{2}+A_{n-1} \alpha+A_{n} .
$$

La seconde dérivée sera égale à

$$
\begin{aligned}
\frac{d^{2} \mathbf{P}_{n}}{d a^{2}}=n(n-1) a^{n-2} & +(n-1)(n-2) \mathbf{A}_{1} a^{n-3}+(n-2)(n-3) \mathbf{A}_{2} a^{n-1} \\
& +\ldots \ldots \ldots+2 \mathbf{A}_{n-2},
\end{aligned}
$$

et la somme des deux fonctions entières doit se róduire à $a^{n}$;

$$
\begin{aligned}
& \begin{aligned}
a^{n}=\alpha^{n}+A_{1}\left|\begin{array}{r}
a^{n-1}+ \\
+A_{2} \\
+n(n-1)
\end{array}\right| \begin{array}{r}
a^{n-2}+A_{3} \\
+(n-1)(n-2) A_{1}
\end{array} \mid \\
+\ldots \ldots \ldots+A_{n} \\
+2 A_{n-2}
\end{aligned} \mid \\
& \text { d'où l'on déduit }
\end{aligned}
$$

$$
\begin{aligned}
& A_{1}=0 \\
& \text { et par suite } A_{1}=0 \text {, } \\
& \mathrm{A}_{2}+n(n-1)=0 \\
& \text { " } \quad \mathbf{A}_{2}=-n(n-1) \text {, } \\
& A_{a}+(n-1)(n-2) A_{1}=0 \\
& A_{s}=0 \text {, } \\
& A_{1}+(n-2)(n-3) A_{2}=0 \\
& \text { " } \\
& A_{4}=n(n-1)(n-2)(n-3) \text {, } \\
& A_{n}+2 A_{n-2}=0 . \\
& A_{b}=0 \text {, }
\end{aligned}
$$


Les coefficients de rang pair $A_{1}, A_{3}, A_{5} \ldots$ sont tous nuls, et l'on a par conséquent

$$
\mathbf{A}_{n-1}=0 \text { si } n \text { est pair, } \mathbf{A}_{n}=0 \text { si } n \text { est impair. }
$$

Les signes des coefficients $\mathbf{A}_{2}, \mathbf{A}_{4}, \mathbf{A}_{6}, \ldots$ sont alternativement - et + , de sorte qu'on a

$$
\begin{aligned}
& \mathbf{A}_{n}=(-1)^{m} \times(n !), \quad \mathbf{A}_{n-1}=0, \quad \text { si } n=2 m ; \\
& \mathbf{A}_{n-1}=(-1)^{m} \times(n !), \quad \mathbf{A}_{n}=0, \quad \text { si } n=2 m+1 \text {. }
\end{aligned}
$$

Soit par exemple $n=6$. On aura

et pour $n=7$

$$
\begin{aligned}
& \mathbf{A}_{2}=-6.5=-30, \quad \mathbf{A}_{1}=\mathbf{A}_{3}=\mathbf{A}_{5}=0, \\
& \mathbf{A}_{4}=+6.5 .4 \cdot 3=360, \\
& \mathbf{A}_{6}=-6.5 .4 .3 .2 \cdot 1=720 ;
\end{aligned}
$$

$$
\begin{aligned}
& A_{2}=-7.6=-42, \quad A_{1}=A_{3}=A_{6}=A_{7}=0, \\
& A_{4}=840, \quad A_{6}=-5040 .
\end{aligned}
$$

Cette règle suffit à la rigueur pour écrire immédiatement les facteurs $\mathbf{P}_{n}$ et $\mathbf{Q}_{n}$ de sina et de cosa. Mais on peut encore la simplifier, grâce aux remarques suivantes.

Nous venons de trouver

(4) $\mathbf{P}_{n}=a^{n}-n(n-1) a^{n-2}+n(n-1)(n-2)(n-3) \alpha^{n-4}-\ldots \ldots$

et en prenant la première dérivée du polynome on en déduit

$\mathrm{Q}_{n}=\frac{d \mathrm{P}_{n}}{d \alpha}=n a^{n-1}-n(n-1)(n-2) \alpha^{n-3}+n(n-1)(n-2)(n-3)(n-4) a^{n-5}$

En comparant ces deux polynomes terme par terme, on reconnaît que $n a^{n-1}$ est la dérivée de $a^{n}$; de sorte que le premier terme de $\mathbf{Q}_{n}$ s'obtient par dérivation du premier terme de $\mathbf{P}_{n}$;

que $n(n-1) a^{n-2}$, second terme de $P_{n}$ changé de signe, est la dérivée de $n \alpha^{n-1}$, premier terme de $\mathbf{Q}_{n}$;

que $n(n-1)(n-2) a^{n \rightarrow 3}$ est la dérivée de $n(n-1) a^{n-2}$, de sorte que la dérivation du second terme de $P_{n}$ donne le second terme de $Q_{n}$,

et ainsi de suite alternativement, en ayant soin d'alterner les signes des termes obtenus, de manière à prendre négativement dans chaque développement les termes de rang pair, positivement les termes de rang impair. 
Pour opérer les développements des deux polynomes, il convient d'écrire alternativement les dérivées formant chaque terme suivant deux lignes horizontales, l'une qui donnera le développement de $P_{n}$, l'autre le développement de $\mathbf{Q}_{n}$ :

$$
\begin{aligned}
& a^{n} \quad n(n-1) a^{n-2} \quad n(n-1)(n-2)(n-3) a^{n-4} \\
& n a^{n-1} \quad n(n-1)(n-2) a^{n-3} n(n-1)(n-2)(n-3)(n-4) a^{n-5}
\end{aligned}
$$

et ainsi de suite; en mettant le signe - aux termes de rang pair dans chaque ligne, il vient en définitive

$$
\begin{aligned}
& \mathrm{P}_{n}=a^{n}-n(n-1) a^{n-2}+n(n-1)(n-2)(n-3) a^{n-4}-\ldots \ldots \\
& \mathrm{Q}_{n}=n a^{n-1}-n(n-1)(n-2) a^{n-3}+n(n-1)(n-2)(n-3)(n-4) a^{n-5}-\ldots
\end{aligned}
$$

Posons par exemple $n=7$. Il viendra

$$
\begin{aligned}
& P_{7}=a^{7}-42 a^{5}+840 a^{3}-5040 a, \\
& Q_{7}=7 a^{8}-210 a^{4}+2520 a^{2}-5040,
\end{aligned}
$$

et l'on aura par conséquent

$$
\begin{aligned}
\int a^{7} \cos a d a= & \left(a^{7}-42 \alpha^{5}+840 a^{3}-5040 a\right) \sin \alpha \\
& +\left(7 a^{6}-210 a^{4}+2520 a^{2}-5040\right) \cos a .
\end{aligned}
$$

$\mathrm{Si}$, au lieu de mettre en facteur les polynomes $\mathrm{P}_{n}, Q_{n}$ qui multiplient respectivement $\sin \alpha$ et $\cos \alpha$, on ordonne le second membre par rapport aux puissances descendantes de $\alpha$, on écrira

$$
\begin{aligned}
\int a^{7} \cos a d a= & a^{7} \sin \alpha+7 a^{6} \cos \alpha-42 \alpha^{5} \sin \alpha-210 a^{4} \cos \alpha \\
& +840 a^{3} \sin \alpha+2520 a^{2} \cos \alpha-5040 \alpha \sin \alpha-5040 \cos \alpha,
\end{aligned}
$$

et il est clair que pareille disposition est applicable au cas général :

(6) $\int a^{n} \cos \alpha d \alpha=a^{n} \sin \alpha+n a^{n-1} \cos \alpha-n(n-1) a^{n-2} \sin \alpha$

$$
-n(n-1)(n-2) a^{n-3} \cos a+\ldots \ldots
$$

Or pour passer d'un terme au suivant, il suffit de prendre séparément les dérivées des deux facteurs qui composent le terme considéré, savoir le monôme contenant la puissance de a et le facteur trigonométrique qui la multiplie; par exemple, si l'on isole les facteurs

$$
a^{n} \text { et } \sin \alpha
$$

qui forment le premier terme, on obtiendra le second en prenant leurs dérivées respectives

$$
n a^{n-1} \text { et } \cos a \text {, }
$$


ce qui donnera

$n a^{n-1} \cos \alpha$;

pour passer au suivant, on isolera de même

$$
n a^{n-1} \text { et } \cos a
$$

et prenant les dérivées de chacun des facteurs on aura

dont le produit est

$$
n(n-1) a^{n-2} \text { et }-\sin a
$$

$$
-n(n-1) a^{n-2} \sin a,
$$

c'est à dire, le troisième terme.

Le quatrième sera de même

$$
\begin{aligned}
& n(n-1)(n-2) a^{n-8} \times(-\cos a) \\
= & -n(n-1)(n-2) a^{n-3} \cos a,
\end{aligned}
$$

et ainsi de suite, jusqu' à épuisement des dérivées des puissances de. $\alpha$; les facteurs trigonométriques règlent le signe de chaque terme, par l'opération même de la dérivation.

Exemple. Écrire l'intégrale $\int \alpha^{10} \cos a d \alpha$.

\begin{tabular}{|c|c|c|}
\hline$a^{10}$ & $\sin \alpha$ & $\int a^{10} \cos a d a=a^{10} \sin a$ \\
\hline $10 a^{9}$ & $\cos a$ & $+10 a^{0} \cos a$ \\
\hline $90 a^{8}$ & $-\sin a$ & $-90 a^{8} \sin \alpha$ \\
\hline $720 a^{7}$ & $-\cos a$ & $-720 a^{7} \cos \alpha$ \\
\hline $5040 a^{6}$ & $\sin a$ & $+5040 \alpha^{6} \sin \alpha$ \\
\hline $30240 a^{5}$ & $\cos a$ & $+30240 a^{6} \cos \alpha$ \\
\hline $151200 a^{4}$ & $-\sin a$ & $-151200 \alpha^{4} \sin \alpha$ \\
\hline $604800 a^{3}$ & $-\cos a$ & $-604800 \alpha^{2} \cos \alpha$ \\
\hline $1814400 a^{2}$ & $\sin a$ & $+1814400 a^{2} \sin a$ \\
\hline $3628800 a$ & $\cos \alpha$ & $+3628800 a$ cosa \\
\hline 3628800 & $-\sin a$ & -3628800 yina \\
\hline
\end{tabular}

On pourra disposer les calculs en trois colonnes verticales, l'une renfermant le monome $a^{10}$ et ses dérivées successives, l'autre sina et ses dérivées, la troisième le produit des dérivées correspondantes prises dans les deux colonnes. 
On aurait du mème coup $P_{10}$ et $Q_{10}$ en ordonnant l'intégrale obtenue par rapport à $\sin \alpha$ et à cosa, et en prenant pour $P_{10}$ le coefficient de sina, pour $Q_{10}$ le coefficient de cosa, ce qui revient à prendre pour $P_{10}$ les termes inscrits dans la première colonne à gauche, de deux en deux en alternant les signes, et pour $\mathbf{Q}_{10}$ les autres termes avec signes alternatifs :

$$
\begin{aligned}
& P_{10}=a^{10}-90 a^{8}+5040 a^{8}-151200 a^{4}+1814400 a^{2}-3628800, \\
& Q_{10}=10 a^{9}-720 a^{7}+30240 a^{5}-604800 a^{3}+3628800 a .
\end{aligned}
$$

On trouverait de même l'intégrale $\int a^{n} \sin \alpha d a$. Posons, par analogie avec ce que nous avons fait pour l'intégrale $\int a^{n} \cos \alpha d a$,

$$
\int \alpha^{n} \sin a d a=M \cos \alpha+N \sin \alpha,
$$

$M$ et $\mathbf{N}$ désignant des polynomes entiers en a, qu'il s'agit de déterminer.

Nous aurons, en différentiant et en divisant par $d a$,

$$
\begin{aligned}
a^{n} \sin \alpha & =-M \sin \alpha+N \cos a+\frac{d M}{d \alpha} \cos \alpha+\frac{d N}{d \alpha} \sin \alpha \\
& =\left(\frac{d N}{d \alpha}-M\right) \sin \alpha+\left(\frac{d M}{d \alpha}+N\right) \cos \alpha
\end{aligned}
$$

d'où ressultent les relations qui assurent l'identité des deux membres :

$$
\left\{\begin{array}{l}
\frac{d \mathrm{~N}}{d a}-\mathrm{M}=a^{n} \\
\frac{d \mathrm{M}}{d a}+\mathrm{N}=0 .
\end{array}\right.
$$

Comparons le système d'équations (9) au système (2):

(2) $\left\{\begin{array}{l}\frac{d P_{n}}{d a}-Q_{n}=0, \\ \frac{d Q_{n}}{d a}+P_{n}=a^{n} .\end{array}\right.$ 
On ramène le premier système au second en posant

$$
\mathbf{N}=\mathbf{Q}_{n} \text { et } \mathbf{M}=-\mathbf{P}_{n},
$$

car la première du groupe (9) devient identique à la seconde du groupe (2), et la seconde du groupe (9) reproduit la première du groupe (2) changée de signe. On aura donc, sans nouveaux calculs, (10) $\int \alpha^{n} \sin a d \alpha=-P_{n} \cos \alpha+Q_{n} \sin \alpha$.

Si l'on rapproche cette formule de notre formule (1)

(1) $\int \alpha^{n} \cos \alpha d a=P_{n} \sin \alpha+Q_{n} \cos \alpha$,

on pourra les fondre en une seule de deux manières, soit en posant

(11) $\int \alpha^{n}\left|\begin{array}{l}\cos \alpha \\ \sin \alpha\end{array}\right| d a=P_{n}\left|\begin{array}{r}\sin \alpha \\ -\cos \alpha\end{array}\right|+Q_{n}\left|\begin{array}{l}\cos \alpha \\ \sin \alpha\end{array}\right|$

ce qui revient à changer $\cos \alpha$ en $\sin \alpha$ et $\sin \alpha$ en $-\cos \alpha$ pour passer de l'équation (1) à l'équation (10), ou en multipliant l'équation (10) par l'unité imaginaire $i=\sqrt{-1}$, et en l'ajoutant ensuite à l'équation (1); il vient en effet

$$
\begin{aligned}
& \int a^{n}(\cos a+i \sin \alpha) d a=\int a^{n} e^{\alpha i} d a \\
& =\mathrm{P}_{n}(\sin \alpha-i \cos \alpha)+\mathrm{Q}_{n}(\cos \alpha+i \sin \alpha) \\
& =\mathrm{P}_{n} \frac{e^{\alpha i}}{i}+\mathrm{Q}_{n} e^{a i}=e^{a i}\left(\mathrm{Q}_{n}-i \mathrm{P}_{n}\right) .
\end{aligned}
$$

\section{$\S 3$}

Nous avons obtenu les relations générales

(4) $\mathbf{P}_{n}=a^{n}-n(n-1) a^{n-2}+n(n-1)(n-2)(n-3) a^{n-4} \ldots \ldots$,

(5) $\mathrm{Q}_{n}=n a^{n-1}-n(n-1)(n-2) a^{n-3}+n(n-1)(n-2)(n-3)(n-4) \alpha^{n-5} \ldots$

Changeons $n$ en $n-1$ dans la première équation; il viendra

$$
P_{n-1}=a^{n-1}-(n-1)(n-2) a^{n-3}+(n-1)(n-2)(n-3)(n-4) a^{n-5} . .
$$


et cette équation multipliée par $n$ reproduit la valeur de $Q_{n}$. On a donc

(14) $\mathrm{Q}_{n}=n \mathrm{P}_{n-1}$,

relation générale qui donne, en y changeant $n$ en $n-1$,

$$
Q_{n-1}=(n-1) \mathrm{P}_{n-2} \text {. }
$$

On a d'ailleurs

$$
\frac{d \mathrm{P}_{n-1}}{d \alpha}=\mathrm{Q}_{n-1}=(n-1) \mathrm{P}_{n-2},
$$

et multipliant par $n$, il vient

$$
n \frac{d \mathrm{P}_{n-1}}{d a}=n(n-1) \mathrm{P}_{n-2}
$$

Formons $\frac{d \mathrm{P}_{n-1}}{d a}$; nous aurons en multipliant par $\boldsymbol{n}$ la dérivée de l'équation (4)

$$
\begin{aligned}
n \frac{d \mathbf{P}_{n-1}}{d a}=n(n-1) a^{n-2}- & n(n-1)(n-2)(n-3) a^{n-4} \\
& +n(n-1)(n-2)(n-3)(n-4)(n-5) a^{n-6} \ldots \ldots,
\end{aligned}
$$

ce qui reproduit, changés de signes, les termes de $P_{n}$ à partir du second. On a donc l'équation

$$
\mathbf{P}_{n}=a^{n}-n \frac{d P_{n-1}}{d a}=a^{n}-n(n-1) P_{n-2}
$$

qui établit une relation de recurrence reliant entre elles les fonctions $P_{n}$ de deux en deux.

On prouverait de même, et on établirait du reste, soit en dérivant l'équation (15), soit en nous servant de l'équation (14),

(16) $Q_{n}=n \alpha^{n-1}-n(n-1) Q_{n-2}$.

Les relations (15) et (16) permettent de former de proche en proche les fonctions $P$ et les fonctions $Q$. On partira de l'intégrale connue $\int \cos \alpha d \alpha=\sin \alpha$ qui montre que $P_{0}=1$ et $Q_{0}=0$; puis de 
l'intégrale $\int \alpha \cos a d a=a \sin a+\cos a$, ce qui entraîne $P_{1}=\alpha, Q_{1}=1$, et l'on formera les deux suites :

$$
\begin{aligned}
& P_{0}=1 \text {, } \\
& P_{2}=a^{2}-2 \text {, } \\
& P_{4}=a^{4}-12\left(a^{2}-2\right)=a^{4}-12 a^{2}+24, \\
& P_{6}=a^{6}-30\left(a^{4}-12 \alpha^{2}+24\right) \\
& =a^{n}-30 a^{4}+360 a^{2}-720 \text {, } \\
& P_{8}=a^{8}-56\left(a^{6}-30 a^{4}+360 a^{2}-720\right) \\
& =a^{8}-56 a^{6}+1680 a^{4}-20160 a^{2}+40320 \text {, } \\
& P_{1}=a, \\
& P_{3}=a^{3}-6 a \text {, } \\
& P_{5}=a^{5}-20\left(a^{3}-6 a\right)=a^{5}-20 a^{3}+120 a \text {, } \\
& P_{7}=a^{7}-42\left(a^{5}-20 a^{3}+120 a\right) \\
& =a^{7}-42 a^{5}+840 a^{3}-5040 a,
\end{aligned}
$$

La série des valeurs de $Q_{n}$ s'obtiendrait de même en partant des relations

$$
Q_{0}=0 \text { et } Q_{1}=+1
$$

et du premier terme de la formule, toujours égal a $n a^{n-1}$.

Il vient

$$
\begin{aligned}
& Q_{2}=2 a, \\
& Q_{4}=4 a^{8}-12 \times 2 a=4 a^{3}-24 a, \\
& Q_{0}=6 a^{5}-30\left(4 a^{3}-24 a\right)=6 a^{5}-120 a^{8}+720 a, \\
& \cdots \cdots \cdots \cdots \cdots \cdots \cdots \cdots \cdots \cdots \cdots \cdots \cdots \cdots \cdots \cdots \\
& Q_{3}=3 a^{2}-6, \\
& Q_{5}=5 a^{4}-20\left(3 a^{2}-6\right)=5 a^{4}-60 a^{2}+120, \text { etc. }
\end{aligned}
$$

